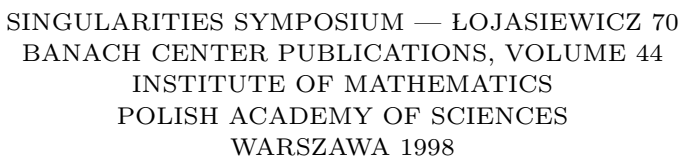

\title{
ON BLOWING UP VERSAL DISCRIMINANTS
}

\author{
PIOTR JAWORSKI \\ Institute of Mathematics, University of Warsaw \\ Banacha 2, 02-097 Warszawa, Poland \\ E-mail: jwptxa@mimuw.edu.pl
}

\begin{abstract}
It is well-known that the versal deformations of nonsimple singularities depend on moduli. The first step in deeper understanding of this phenomenon is to determine the versal discriminant, which roughly speaking is an obstacle for analytic triviality of an unfolding or deformation along the moduli.

The goal of this paper is to describe the versal discriminant of $Z_{k, 0}$ and $Q_{k, 0}$ singularities basing on the fact that the deformations of these singularities may be obtained as blowing ups of certain deformations of $J_{k, 0}$ singularities.
\end{abstract}

\section{Introduction. Let}

$$
f:\left(\mathbb{C}^{n}, 0\right) \rightarrow(\mathbb{C}, 0)
$$

be a germ of an analytic function with an isolated critical point at the origin. Let

$$
F: U \rightarrow \mathbb{C}, \quad(0,0) \in U \subset \mathbb{C}^{n} \times \Lambda
$$

be an analytic deformation of $f\left(f(x)=F(x, 0)_{0}\right)$, which is miniversal for right equivalence. Obviously $F_{\lambda}(x)=F(x, \lambda)$ is versal for V-equivalence (contact equivalence).

Let $T$ be the moduli set, i.e. the subset of $\Lambda$ consisting of such $\lambda$ that $F_{\lambda}(x)$ has a critical point $p$ of multiplicity $\mu=\mu(f)$ and $F_{\lambda}(p)=0$.

Assume that $T$ is smooth. Let $\pi:(\Lambda, 0) \rightarrow(T, 0)$ be an analytic projection (transversal to $T$ ). The versal discriminant $V$ of the deformation $F_{\lambda}$ relative to the projection $\pi$ is the subset of the fiber $\pi^{-1}(0)$ consisting of such parameters $\lambda$ that the deformation

$$
F_{\lambda}: \lambda \in \pi^{-1}(0)
$$

is not infinitesimally $\mathrm{V}$-versal at $\lambda$ (i.e. it is not transversal to the strata of $\mathrm{V}$-equivalence).

Now let the germ $f$ be quasihomogeneous (weighted homogeneous). Then there exists a distinguished class of projections induced by quasihomogeneity. Indeed, let $v$ be an associated quasihomogeneous weight. We consider the quasihomogeneous miniversal

1991 Mathematics Subject Classification: Primary 32S15; Secondary 32G13, 14 B07.

Received by the editors: January 10, 1997.

The paper is in final form and no version of it will be published elsewhere. 
deformation:

$$
F_{\lambda}=f+\sum_{i=1}^{\mu} \lambda_{i} e_{i}
$$

where $e_{1}, \ldots, e_{\mu}$ is a quasihomogeneous base of the local algebra $\mathcal{O}_{n} / I_{f}$, where $I_{f}$ is the ideal spanned by partial derivatives $\partial f / \partial x_{i} i=1, \ldots, n$ (compare [1] $\S 8$ ). We say that the parameter $\lambda_{i}$ is under-, over-, or diagonal if the weight of $e_{i}$ is less than, greater than or equal to the weight of $f$. In this case the moduli set $T$ is a linear subspace of the base $\Lambda=\mathbb{C}^{\mu}$ spanned by over- and diagonal $\lambda$ 's. Moreover, there is a canonical projection $\pi$ onto $T$ - "forgetting" the underdiagonal $\lambda$ 's. For such a projection the restriction $F_{\lambda}$, $\lambda \in \pi^{-1}(0)$ is a part of the deformation consisting of underdiagonal terms, so called underdiagonal deformation (called also the deformation of negative weight). Since the quasihomogeneous germs are germs of polynomials, $F_{\lambda}$ is defined globally and we may put for example $U=\mathbb{C}^{n} \times \mathbb{C}^{\mu}$. Nevertheless since the versal discriminant depends on the choice of the domain where the deformation is defined, we restrict ourselves to the domains $U$ such that:

- $U$ splits; $U=\left\{\left(x, \lambda_{1}, \lambda^{\prime}\right): \lambda_{1} \in \mathbb{C},\left(x, \lambda^{\prime}\right) \in U^{\prime}\right\}$,

- $U$ is a neighborhood of $\mathbb{C}^{n} \times \pi^{-1}(0)$,

- the factor algebra of analytic functions on $U$ modulo the ideal generated by partial derivatives $\partial F_{\lambda} / \partial x_{i}, i=1, \ldots, n$ is a free module over the ring of analytic functions on $\Pi(U)$ (the projection of $U$ on $\Lambda$ ) generated by introduced above polynomials $e_{1}, \ldots, e_{\mu}$, i.e.

$$
\mathcal{O}(U) / I_{F_{\lambda}}=\mathcal{O}(\Pi(U)) \otimes_{\mathbb{C}} \mathcal{O}_{n} / I_{f}
$$

In this paper we apply the results concerning the $J_{k, 0}$ singularities (see [7]) and describe the versal discriminants, relative to the canonical projections onto the moduli sets, of quasihomogeneous miniversal deformations of $Z_{k, 0}$ and $Q_{k, 0}$ singularities, restricted to the domains $U$ as above.

We consider the deformations of $J_{k, 0}$ singularities with a critical point (resp. degenerated critical point) at the origin. Next we blow up the origin. The strict transformation of such a family of functions shows to be a deformation of $Z_{k, 0}$ (resp. $Q_{k, 0}$ ). Moreover we obtain the whole miniversal deformations in such a way. Furthermore the versal discriminant is mapped to the versal discriminant.

We would like to mention here that the notions similar to the versal discriminant (versality discriminant, instability locus, ...) were investigated by many authors. The reader is referred for example to works of J. Damon or A. du Plessis and C. T. C. Wall$[2,3,4,9,10]$ or to the other papers on topological triviality- $[8,11]$.

2. Notation. We recall the basic definitions. Let $F(x, \lambda)$ be an analytic deformation of $f(x)$ defined on a domain $U$. An analytic vector field $\eta$ defined on $\Pi(U)$ is called liftable if there exists an analytic vector field $\xi$ defined on $U$ such that

$$
\xi=\sum \xi_{i}(x, \lambda) \frac{\partial}{\partial x_{i}}+\eta
$$

and $\xi(F)=A F$, where $A(x, \lambda)$ is analytic. We call such a vector field $\xi$ a lifting of $\eta$. Obviously the lifting is tangent to the zero set of $F$. 
We shall denote by $\mathcal{M}$ the $\mathcal{O}(\Pi(U))$ module of analytic liftable vector fields and by $\mathcal{M}(\lambda)$ the $\mathbb{C}$-linear space of their values at the point $\lambda$.

We recall that the tangent space to a $\mathrm{V}$-equivalence stratum is spanned by liftable vector fields. Therefore we apply the following definition (compare the notion of a versality discriminant $[2,3,4]$ and an instability locus [9]):

A point $\lambda \in \pi^{-1}(0)$ belongs to the versal discriminant relative to the projection $\pi$ if and only if the module of analytic liftable vector fields $\mathcal{M}$ is not transversal to the fiber $\pi^{-1}(0)$ at $\lambda$

$$
\mathcal{M}(\lambda) \oplus T_{\lambda} \pi^{-1}(0) \neq T_{\lambda} \Lambda
$$

3. The main results. In this paper we consider the quasihomogeneous analytic functions

$$
f(x, y)=x y^{3}+\beta y x^{2 k-1}+\gamma x^{3 k-2}, \quad k=2,3, \ldots,
$$

and

$$
g(x, y, z)=x z^{2}+y^{3}+\beta y x^{2 k-2}+\gamma x^{3 k-3} \quad k=2,3, \ldots,
$$

where $4 \beta^{3}+27 \gamma^{2} \neq 0$.

They have an isolated singular point at the origin. In Arnold's classification (see [1] §15) such singularities are called respectively $Z_{k-2,0}$ and $Q_{k-1,0}$ for $k>2$ and $X_{9}$ and $Q_{8}$ for $k=2$. To simplify statements we put $X_{9}=Z_{0,0}$ and $Q_{8}=Q_{1,0}$. We remark that the above singularities are classified by $j$-invariant:

$$
j=\frac{4 \beta^{3}}{4 \beta^{3}+27 \gamma^{2}},
$$

the same which classifies the elliptic curves

$$
z^{2}=(y+\alpha x)^{3}+\beta(y+\alpha x) x^{2}+\gamma x^{3}
$$

(compare [5] $\S \mathrm{IV} .4$ ), therefore they can be described by normal forms which depend only on one diagonal modulus.

Our aim is to describe the versal discriminant of these singularities relative to the projection $\pi$ onto over- and diagonals;

$$
\pi\left(\lambda_{\text {underdiagonal }}, \lambda_{\text {diagonal }}, \lambda_{\text {overdiagonal }}\right)=\left(\lambda_{\text {diagonal }}, \lambda_{\text {overdiagonal }}\right) .
$$

It shows that the versal discriminants do not depend on the choice of the particular quasihomogeneous deformation. Furthermore, with the exception when $k \geq 3$ and the singularity is harmonic or aharmonic $(j=0,1)$, the topological type of the versal discriminant does not depend on the $j$-invariant.

Let $F(x, y, \lambda)$ and $G(x, y, z, \lambda)$ be miniversal quasihomogeneous deformations of $f$ and $g$.

THEOREM 1. The versal discriminant of $Z_{k-2,0}, k=2,3, \ldots$, consists of all parameters $\lambda$ such that, after a substitution $y=y-a(x), F_{\lambda}$ is one of the following polynomials:
$\mathrm{A}: x y^{3}+e x^{2 m-1} d(x)^{2} y+x^{3 m-2} d(x)^{3}$,
B: $x y^{3}+x^{m-1} b(x) y$
$m=1,2, \ldots, k$,
C: $x y^{3}+x^{m-2} c(x)$,
$m=1,2, \ldots, 2 k$,
$m=2,3, \ldots, 3 k$, 
where $27+4 e^{3} \neq 0 ; b(x), c(x), d(x)$ are polynomials of degree respectively $2 k-m, 3 k-m$, $k-m$ with no more than $k-2$ different nonzero roots; $a(x)$ is any polynomial of degree $k-1$ or less.

TheOREM 2. The versal discriminant of $Q_{k-1,0}, k=2,3, \ldots$, consists of all parameters $\lambda$ such that, after a substitution $y=y-a(x), G_{\lambda}$ is one of the following polynomials:
A: $x z^{2}+y^{3}+e x^{2 m-2} d(x)^{2} y+x^{3 m-3} d(x)^{3}$
B: $x z^{2}+y^{3}+x^{m-2} b(x) y$,
$m=1,2, \ldots, k$
C: $x z^{2}+y^{3}+x^{m-3} c(x)$,
$m=2,3 \ldots, 2 k$
$\mathrm{D}: x z^{2}+2 a z+y^{3}+c_{1}(x)$,
$m=3,4, \ldots, 3 k$,
$a \neq 0$,

where $27+4 e^{3} \neq 0 ; b(x), c(x), d(x), x c_{1}(x)-a^{2}$ are polynomials of degree respectively $2 k-m, 3 k-m, k-m, 3 k-2$ with no more than $k-2$ different nonzero roots; $a(x)$ is any polynomial of degree $k-1$ or less.

Remark 1. The cases A, B, C, D from the above theorems occur for the quasihomogeneous singularities with $j$-invariant equal respectively to $27 /\left(27+4 e^{3}\right), 1,0,0$.

4. Blowing down the deformations. The goal of this section is to describe the link between deformations of $J_{k, 0}$ singularities and $Z_{k-2,0}$ and $Q_{k-1,0}$. In order to limit the number of formulas we introduce two parameters $\hat{\gamma}$ and $\hat{\beta}$ :

$$
(\hat{\gamma}, \hat{\beta})= \begin{cases}(0,1) & \text { if } \gamma=0 \\ (1,0) & \text { if } \gamma \neq 0\end{cases}
$$

4.1. $Z_{k, 0}$ case. We write down the miniversal deformation of $Z_{k-2,0}$ singularity

$$
f(x, y)=x y^{3}+\beta y x^{2 k-1}+\gamma x^{3 k-2}, \quad 4 \beta^{3}+27 \gamma^{2} \neq 0, \quad k=2,3, \ldots,
$$

in a form

$$
F(x, y, \lambda)=f(x, y)+\lambda_{2,0} y^{2}+\sum_{i=0}^{3 k-3} \lambda_{0, i} x^{i}+\sum_{i=0}^{2 k-2} \lambda_{1, i} y x^{i}+\sum_{i=0}^{k-2} \lambda_{e, i}\left(\hat{\gamma} y+\hat{\beta} x^{k-1}\right) x^{2 k+i-1}
$$

where $(x, y, \lambda) \in \mathbb{C}^{2} \times \mathbb{C}^{6 k-3}$ (compare [1] $\S 8.2$ and $\S 8.3$ ).

Next we blow down the line $x=0$. We put

$$
h(x, y)=x^{2} f(x, y / x), \quad F_{1}(x, y, \lambda)=x^{2} F(x, y / x, \lambda) .
$$

$h$ has a $J_{k, 0}$ singularity at the origin and $F_{1}$ is its deformation. Indeed:

$$
\begin{gathered}
h(x, y)=y^{3}+\beta y x^{2 k}+\gamma x^{3 k}, \\
F_{1}(x, y, \lambda)=h(x, y)+\lambda_{2,0} y^{2}+\sum_{i=2}^{3 k-1} \lambda_{0, i-2} x^{i}+\sum_{i=1}^{2 k-1} \lambda_{1, i-1} y x^{i}+\sum_{i=0}^{k-2} \lambda_{e, i}\left(\hat{\gamma} y+\hat{\beta} x^{k}\right) x^{2 k+i}
\end{gathered}
$$

where $(x, y, \lambda) \in \mathbb{C}^{2} \times \mathbb{C}^{6 k-3}$. We remark that all functions $F_{1}(\cdot, \cdot, \lambda)$ have a critical point at the origin and the corresponding critical value is 0 .

$F_{1}$ as every other deformation is equivalent to the one induced from the miniversal. If we select the miniversal deformation of $h$ in a form

$$
H(x, y, \tau)=h(x, y)+\sum_{i=0}^{3 k-2} \tau_{0, i} x^{i}+\sum_{i=0}^{2 k-2} \tau_{1, i} y x^{i}+\sum_{i=0}^{k-1} \tau_{e, i}\left(\hat{\gamma} y+\hat{\beta} x^{k}\right) x^{2 k+i-1}
$$


where $(x, y, \tau) \in \mathbb{C}^{2} \times \mathbb{C}^{6 k-2}$, then $F_{1}$ is induced by a shift

$$
\begin{aligned}
& x=x^{\prime}-x_{0}, \quad y=y^{\prime}-y_{0}, \\
& x_{0}= \begin{cases}\frac{-\lambda_{0,3 k-3}}{3 k \gamma} & \text { if } \gamma \neq 0 \\
\frac{-\lambda_{1,2 k-2}}{2 k \beta} & \text { if } \gamma=0,\end{cases} \\
& y_{0}=\frac{1}{3} \lambda_{2,0},
\end{aligned}
$$

i.e.

$$
F_{1}(x, y, \lambda)=H\left(x+x_{0}, y+y_{0}, \varphi(\lambda)\right) .
$$

We remark that $\varphi$ is a polynomial mapping whose image coincides with the set of parameters $\tau$ such that the curve $H_{\tau}=0$ has a singular point. Since its domain is smooth and it is injective in regular points, $\varphi$ is a normalization of its image.

4.2. $Q_{k, 0}$ case. We write down the miniversal deformation of $Q_{k-1,0}$ singularity

$$
g(x, y)=x z^{2}+y^{3}+\beta y x^{2 k-2}+\gamma x^{3 k-3}, \quad 4 \beta^{3}+27 \gamma^{2} \neq 0, \quad k=2,3, \ldots,
$$

in a form

$$
\begin{aligned}
G(x, y, \lambda)=g(x, y, z)+ & \lambda_{1,1,0} y z+\lambda_{1,0,0} z \\
& +\sum_{i=0}^{3 k-4} \lambda_{0, i} x^{i}+\sum_{i=0}^{2 k-3} \lambda_{1, i} y x^{i}+\sum_{i=0}^{k-2} \lambda_{e, i}\left(\hat{\gamma} y+\hat{\beta} x^{k-1}\right) x^{2 k+i-2}
\end{aligned}
$$

where $(x, y, z, \lambda) \in \mathbb{C}^{3} \times \mathbb{C}^{6 k-4}$.

First we blow down lines $x=0, y=$ const. The transform of $g$ is $f+z^{2}$, indeed

$$
x g\left(x, y, z x^{-1}\right)=z^{2}+x y^{3}+\beta y x^{2 k-1}+\gamma x^{3 k-2} .
$$

We put

$$
\begin{array}{rl}
G_{1}(x, y, z, \lambda)=x & G\left(x, y, z x^{-1}, \lambda\right)=z^{2}+f(x, y)+\lambda_{1,1,0} y z+\lambda_{1,0,0} z \\
+\sum_{i=1}^{3 k-3} \lambda_{0, i-1} x^{i}+\sum_{i=1}^{2 k-2} \lambda_{1, i-1} y x^{i}+\sum_{i=0}^{k-2} \lambda_{e, i}\left(\hat{\gamma} y+\hat{\beta} x^{k-1}\right) x^{2 k+i-1}
\end{array}
$$

where $(x, y, z, \lambda) \in \mathbb{C}^{3} \times \mathbb{C}^{6 k-4}$. $G_{1}$ is induced from $z^{2}+F$ by a shift

$$
z=z^{\prime}-\frac{1}{2}\left(\lambda_{1,1,0} y+\lambda_{1,0,0} x\right)
$$

Thus

$$
G_{1}(x, y, z, \lambda)=F\left(x, y, \varphi_{1}(\lambda)\right)+\left(z+\frac{1}{2}\left(\lambda_{1,1,0} y+\lambda_{1,0,0} x\right)\right)^{2} .
$$

The image of $\varphi_{1}$ consists of such parameters $\lambda$ that the curve $F_{\lambda}=0$ is tangent to the axis $x=0$ or contains it. Furthermore $\varphi_{1}$ is a branched double covering.

Next we blow down the plane $x=0$. Analogically as in the previous subsection the transform of $f+z^{2}$ is $h+z^{2}$ and of $F+z^{2}$ is $H+z^{2}$. Moreover the image of $\varphi \circ \varphi_{1}$ consists of such parameters $\tau$ that the curve $H_{\tau}=0$ has a degenerated singular point. 
5. Transformations of liftable vector fields. Let $\Phi$ be an analytic fibered morphism

$$
\Phi: X \times \Lambda \longrightarrow Y \times \Omega, \quad \Phi(x, \lambda)=(\bar{\Phi}(x, \lambda), \varphi(\lambda)) .
$$

We shall denote the differential of $\Phi$ in the following way

$$
D \Phi=\left(\begin{array}{cc}
D_{x} \bar{\Phi} & D_{\lambda} \bar{\Phi} \\
0 & D_{\lambda} \varphi
\end{array}\right) .
$$

Furthermore, let $U$ be an open subset of $Y \times \Omega, F: U \longrightarrow \mathbb{C}$ an analytic function, $\eta$ a liftable (with respect to $F$ ) vector field on $\Pi(U)$, and $\xi$ its lifting.

$$
\begin{aligned}
\xi(y, \omega)=\bar{\xi}(y, \omega)+\eta(\omega) & =\sum \bar{\xi}_{i}(y, \omega) \frac{\partial}{\partial y_{i}}+\sum \eta_{i}(\omega) \frac{\partial}{\partial \omega_{i}}, \\
\xi(F)(y, \omega) & =A(y, \omega) F(y, \omega) .
\end{aligned}
$$

We state here several results concerning $D \varphi^{-1}(\eta)$ and $D \Phi^{-1}(\xi)$.

5.1. Stabilization. Assume that $X=Y \times \mathbb{C}, \Lambda=\Omega, \bar{\Phi}$ is a projection $\bar{\Phi}\left(x^{\prime}, x_{n+1}\right)=x^{\prime}$ and $\varphi$ is an identity.

Proposition 3. $\eta$ is liftable with respect to the function $F_{1}(x)=F\left(x^{\prime}\right)+x_{n+1}^{2}$ defined on $U \times \mathbb{C}$ and its lifting equals

$$
\xi_{1}(x, \omega)=\xi\left(x^{\prime}, \omega\right)+A\left(x^{\prime}, \omega\right) \frac{x_{n+1}}{2} \frac{\partial}{\partial x_{n+1}} .
$$

Proof.

$$
\xi_{1}\left(F_{1}\right)=\xi(F)+A \frac{x_{n+1}}{2} \frac{\partial}{\partial x_{n+1}}\left(x_{n+1}^{2}\right)=A F+A x_{n+1}^{2}=A F_{1} .
$$

5.2. Normalization. Assume that $\bar{\Phi}(x, \lambda)$ is a diffeomorphism for a fixed $\lambda$.

Proposition 4. If $\eta_{1}$ is such an analytic vector field that $D_{\lambda} \varphi\left(\eta_{1}\right)=\eta$ then it is liftable with respect to the function $F_{1}=F \circ \Phi$ defined on $U_{1}=\Phi^{-1}(U)$ and its lifting equals

$$
\xi_{1}(x, \lambda)=\left(D_{x} \bar{\Phi}\right)^{-1}\left(\bar{\xi}-D_{\lambda} \bar{\Phi}\left(\eta_{1}\right)\right)+\eta_{1} .
$$

Proof. We have

$$
\begin{aligned}
D \Phi\left(\xi_{1}\right)(F)=\left(D _ { x } \overline { \Phi } \left(\left(D_{x} \bar{\Phi}\right)^{-1}\right.\right. & \left.\left.\left(\bar{\xi}-D_{\lambda} \bar{\Phi}\left(\eta_{1}\right)\right)+D_{\lambda} \bar{\Phi}\left(\eta_{1}\right)\right)+D_{\lambda} \varphi\left(\eta_{1}\right)\right)(F) \\
& =\left(\bar{\xi}-D_{\lambda} \bar{\Phi}\left(\eta_{1}\right)+D_{\lambda} \bar{\Phi}\left(\eta_{1}\right)+\eta\right)(F)=\xi(F)=A F .
\end{aligned}
$$

Thus

$$
\xi_{1}(F \circ \Phi)(x, \lambda)=D \Phi\left(\xi_{1}\right)(F)(\Phi(x, \lambda))=A(\Phi(x, \lambda)) F_{1}(x, \lambda) .
$$

5.3. Blowing up. Assume that $\varphi$ is an identity and $\bar{\Phi}$ is an affine blowing up which does not depend on $\omega$

$$
\Phi\left(x_{1}, x_{2}, \ldots, x_{n}, \omega\right)=\left(x_{1}, x_{2} x_{1}, \ldots, x_{m} x_{1}, x_{m+1}, \ldots, x_{n}, \omega\right) .
$$

Let $F_{1}$ be a strict transform of $F$

$$
F_{1}(x, \omega)=x_{1}^{-p} F \circ \Phi(x, \omega) .
$$

Proposition 5. If $\xi$ is tangent to the center of $\Phi$ then $\eta$ is liftable with respect to $F_{1}$ and its lifting $\xi_{1}$ is tangent to the exceptional divisor. 
Proof. Since $\xi$ is tangent to the center $C=\left\{y_{1}=\ldots=y_{m}=0\right\}$ of the blowing up, $(D \Phi)^{-1}(\xi)$ is analytic. Indeed:

$$
\begin{aligned}
D \Phi\left(\frac{\partial}{\partial x_{1}}\right) & =\frac{\partial}{\partial y_{1}}+\sum_{i=2}^{m} \frac{y_{i}}{y_{1}} \frac{\partial}{\partial y_{i}}, \\
D \Phi\left(\frac{\partial}{\partial x_{i}}\right) & =y_{1} \frac{\partial}{\partial y_{i}}, \quad i=2, \ldots, m, \\
D \Phi\left(\frac{\partial}{\partial x_{i}}\right) & =\frac{\partial}{\partial y_{i}}, \quad i=m+1, \ldots, n .
\end{aligned}
$$

Therefore

$$
\begin{aligned}
& (D \Phi)^{-1}\left(\frac{\partial}{\partial y_{i}}\right)=\frac{\partial}{\partial x_{i}}, \quad i=m+1, \ldots, n, \\
& (D \Phi)^{-1}\left(\frac{\partial}{\partial y_{i}}\right)=\frac{1}{x_{1}} \frac{\partial}{\partial x_{i}}, \quad i=2, \ldots, m, \\
& (D \Phi)^{-1}\left(\frac{\partial}{\partial y_{1}}\right)=\frac{\partial}{\partial x_{1}}-\sum_{i=2}^{m} \frac{x_{i}}{x_{1}} \frac{\partial}{\partial x_{i}}
\end{aligned}
$$

Furthermore, since $\xi$ is tangent to $C=\left\{y_{1}=\ldots=y_{m}=0\right\}$, its coefficients $\overline{\xi_{1}}, \ldots, \overline{\xi_{m}}$ vanish at $C$. Hence

$$
\overline{\xi_{i}}(\Phi(x, \omega))=x_{1} \cdot \hat{\xi}_{i}(x, \omega), \quad i=1, \ldots, m,
$$

where $\hat{\xi}_{i}$ are analytic. We put

$$
\xi_{1}=(D \Phi)^{-1}(\xi)=x_{1} \hat{\xi_{1}} \frac{\partial}{\partial x_{1}}+\sum_{i=2}^{m}\left(\hat{\xi}_{i}-x_{i} \hat{\xi_{1}}\right) \frac{\partial}{\partial x_{i}}+\sum_{i=m+1}^{n} \overline{\xi_{i}} \circ \Phi \frac{\partial}{\partial x_{i}}+\eta .
$$

The first coefficient vanishes at the exceptional divisor $E=\left\{x_{1}=0\right\}$ hence $\xi_{1}$ is tangent to $E$. On the other hand

$$
\xi_{1}\left(x_{1}^{p} F_{1}\right)(x, \omega)=\xi_{1}(F \circ \Phi)(x, \omega)=\xi(F)(\Phi(x, \omega))=A(\Phi(x, \omega)) \cdot x_{1}^{p} F_{1}(x, \omega),
$$

and

Hence

$$
\xi_{1}\left(x_{1}^{p} F_{1}\right)=p x_{1}^{p-1} F_{1} \xi_{1}\left(x_{1}\right)+x_{1}^{p} \xi_{1}\left(F_{1}\right)=p x_{1}^{p} \hat{\xi_{1}} F_{1}+x_{1}^{p} \xi_{1}\left(F_{1}\right) .
$$

$$
\xi_{1}\left(F_{1}\right)=\left(A \circ \Phi-p \hat{\xi_{1}}\right) F_{1}
$$

6. Blowing up a versal discriminant. In this section we will show that the versal discriminant of $Z_{k, 0}$ (resp. $\left.Q_{k}, 0\right)$ is contained in the preimage of the versal discriminant of $J_{k, 0}$.

Let $H(x, y, \tau)$ be a deformation of $J_{k, 0}$ singularity introduced in Subsection 4.1. We remark that due to Proposition 3 every $H(x, y, \tau)$-liftable vector field is also $\left(z^{2}+H(x, y, \tau)\right)$-liftable. Thus both deformations have the same versal discriminant.

We denote by $\Sigma_{1}$ and by $\Sigma_{2}$ subsets of the domain $U$ consisting of such elements $(x, y, \tau)$ that

and

$$
H=0, \quad \nabla H=0
$$

$$
H=0, \quad \nabla H=0, \quad \operatorname{det} D^{2} H=0
$$


and by $\Delta_{1}$ resp. $\Delta_{2}$ their projections, i.e. sets of such parameters $\tau$ that the curve $H_{\tau}=0$ has a singular or resp. degenerated singular point. We remark that every liftable vector field is tangent to both $\Delta_{1}$ and $\Delta_{2}$ and its lifting is tangent to $\Sigma_{1}$ and $\Sigma_{2}$.

6.1. $Z_{k, 0}$ case. Since $\Sigma_{1}$ is described by equations

$$
\begin{aligned}
& 0=\tau_{0,0}+\sum_{i=1}^{3 k-2} \tau_{0, i} x^{i}+\sum_{i=0}^{2 k-2} \tau_{1, i} y x^{i}+\sum_{i=0}^{k-1} \tau_{e, i}\left(\hat{\gamma} y+\hat{\beta} x^{k}\right) x^{2 k+i-1}+h(x, y), \\
& 0=\tau_{0,1}+\sum_{i=2}^{3 k-2} i \tau_{0, i} x^{i-1}+\sum_{i=1}^{2 k-2} i \tau_{1, i} y x^{i-1} \\
& +\sum_{i=0}^{k-1} \tau_{e, i}\left((2 k+i-1) \hat{\gamma} y+(3 k+i-1) \hat{\beta} x^{k}\right) x^{2 k+i-2}+\partial_{x} h(x, y), \\
& 0=\tau_{1,0}+\sum_{i=1}^{2 k-2} \tau_{1, i} x^{i}+\partial_{y} h(x, y),
\end{aligned}
$$

it is a manifold. Furthermore over a generic point of $\Delta_{1}$ lies just one point of $\Sigma_{1}$ hence the projection of $\Sigma_{1}$ onto $\Delta_{1}$ is a normalization. The mapping $\varphi$ introduced in Subsection 4.1 is a normalization as well, therefore, up to the diffeomorphism of normalizations, for any liftable vector field $\eta$ we may put $(D \varphi)^{-1}(\eta)=\xi_{\mid \Sigma_{1}}$. Next, applying Propositions 4 and 5 we show that every $H$-liftable vector field corresponds to the $F$-liftable one. Furthermore the transformation described in 4.1 maps an underdiagonal deformation to an underdiagonal hence the image of a versal discriminant is contained in a versal discriminant.

6.2. $Q_{k, 0}$ case. Since the domain of $\varphi_{1}$ is smooth and the codimension of the set of its critical points is $2,\left(D \varphi_{1}\right)^{-1}$ of any analytic vector field tangent to the image of $\varphi_{1}$ is analytic. This is our case since for every $H$-liftable vector field $\eta,(D \varphi)^{-1}(\eta)$ is tangent to the preimage $(\varphi)^{-1}\left(\Delta_{2}\right)$ which coincides with the image of $\varphi_{1}$.

Next, applying Propositions 4 and 5 we show that every $H$-liftable vector field corresponds to the $G$-liftable one. Furthermore the transformation described in Subsection 4.2 maps an underdiagonal deformation to an underdiagonal hence the image of a versal discriminant is contained in a versal discriminant.

6.3. Conclusions. Let $F, G, H$ be miniversal deformations of respectively $J_{k, 0}, Z_{k, 0}$ and $Q_{k-1,0}$ singularity as in Section 4 . As is shown in [7], the versal discriminant of $J_{k, 0}$ singularity consists of such parameters $\lambda$ that, after a substitution $y=y-a(x), H_{\lambda}$ is one of the following polynomials:

$$
\begin{aligned}
& \mathrm{A}: y^{3}+e d(x)^{2} y+d(x)^{3}, \\
& \mathrm{~B}: y^{3}+b(x) y, \\
& \mathrm{C}: y^{3}+c(x),
\end{aligned}
$$

where $27+4 e^{3} \neq 0 ; b(x), c(x), d(x)$ are polynomials of degree respectively $2 k, 3 k, k$ with no more than $k-1$ different roots; $a(x)$ is any polynomial of degree $k$ or less.

In [6] the reader can find the classification of singular points of zero sets of the above polynomials.

Shifting the roots of respectively $d(x), b(x)$ or $c(x)$ to zero and applying to the above polynomials A, B and C transformations described in Section 4 we obtain 
COROllary 6. The versal discriminant of $Z_{k, 0}$ is contained in a set consisting of such parameters $\lambda$ that, after a substitution $y=y-a(x), F_{\lambda}$ is one of the following polynomials:

$$
\begin{aligned}
& \text { A: } x y^{3}+e x^{2 m-1} d(x)^{2} y+x^{3 m-2} d(x)^{3}, \\
& \text { B: } x y^{3}+x^{m-1} b(x) y, \\
& \text { C: } x y^{3}+x^{m-2} c(x),
\end{aligned}
$$

where $27+4 e^{3} \neq 0 ; b(x), c(x), d(x)$ are polynomials of degree respectively $2 k-m, 3 k-m$, $k-m$ with no more than $k-2$ different nonzero roots; $a(x)$ is any polynomial of degree $k-1$ or less.

COROLlary 7. The versal discriminant of $Q_{k, 0}$ is contained in a set consisting of such parameters $\lambda$ that, after a substitution $y=y-a(x), G_{\lambda}$ is one of the following polynomials:

$$
\begin{aligned}
& \text { A: } x z^{2}+y^{3}+e x^{2 m-2} d(x)^{2} y+x^{3 m-3} d(x)^{3}, \\
& \text { B: } x z^{2}+y^{3}+x^{m-2} b(x) y, \\
& \text { C: } x z^{2}+y^{3}+x^{m-3} c(x), \\
& \text { D: } x z^{2}+2 a z+y^{3}+c_{1}(x), \quad a \neq 0,
\end{aligned}
$$

where $27+4 e^{3} \neq 0 ; b(x), c(x), d(x), x c_{1}(x)-a^{2}$ are polynomials of degree respectively $2 k-m, 3 k-m, k-m, 3 k-3$ with no more than $k-2$ different nonzero roots; $a(x)$ is any polynomial of degree $k-1$ or less.

We describe the last case $\mathrm{D}$ in more details. We start with a polynomial

$$
H=z^{2}+y^{3}+c(x) x^{2}, \quad c(0)=-a^{2} \neq 0 .
$$

After the substitution $z=z^{\prime}+a x$ we obtain

$$
z^{2}+2 a z x+y^{3}+c_{1}(x) x^{2}, \quad c_{1}(x)=\left(c(x)+a^{2}\right) / x .
$$

After weighted blowing up $(x, y, z) \mapsto\left(x, x y, x^{2} z\right)$ we get

$$
x^{3}\left(x z^{2}+2 a z+y^{3}+c_{1}(x)\right) .
$$

From the estimate for $J_{k, 0}$ singularities we know that $c(x) x^{2}$ has no more than $k-1$ different roots. Hence $c(x)=x c_{1}(x)-a^{2}$ has no more than $k-2$ different nonzero roots.

7. Final estimates. To finish the proof of the theorems it is enough to show that the estimate on the number of different nonzero roots of the polynomials $b(x), c(x), d(x)$ and $4 x c_{1}(x)-1$ is strict. We apply the same method as in [7].

Let $e_{1}, \ldots, e_{\mu}$ be a quasihomogeneous basis of the factor algebra $\mathbb{C}[x, y] / I_{F_{\lambda}}$ (resp. $\left.\mathbb{C}[x, y] / I_{G_{\lambda}}\right)$. We remark that a point $\lambda \in \pi^{-1}(0)$ does not belong to the versal discriminant relative to the projection $\pi$ if and only if the polynomials $e_{i} F_{\lambda}$ (resp. $e_{i} G_{\lambda}$ ) and the underdiagonal base elements generate the whole algebra $\mathbb{C}[x, y] / I_{F_{\lambda}}$ (resp. $\left.\mathbb{C}[x, y] / I_{G_{\lambda}}\right)$.

\section{1. $Z_{k, 0}$ case}

LEMma 8. The point $\lambda$ belongs to versal discriminant if and only if $F_{\lambda}$ is of type $A$, $B$ or $C$ and respectively the polynomial $x d(x), x b(x)$, or $x c(x)$ has at most $k-1$ different roots. 
Proof. In $Z_{k, 0}$ case it is enough to consider polynomials $F_{\lambda}, x F_{\lambda}, \ldots, x^{m} F_{\lambda}, \ldots$, $y F_{\lambda}, x y F_{\lambda}, \ldots, x^{m} y F_{\lambda}, \ldots$ and $y^{2} F_{\lambda}$.

We recall the main points of the proof of the $J_{k, 0}$ case (see [7]). The polynomial $H$ (of type A,B or $\mathrm{C}$ ) belongs to the versal discriminant if and only if it fulfils two relations:

- For every $k \geq 0$ there is a polynomial $r_{k}(x)$ such that the polynomial

$$
W_{k}(x, y)=x^{k}\left(3 H(x, y)-y H_{y}(x, y)\right)-r_{k}(x) H_{x}(x, y)
$$

multiplied by $x$ is still underdiagonal, i.e. qdeg $W_{k}<3 k-1$.

- There are two relatively prime polynomials $p(x)$ and $q(x)$ such that degree of $p$ is not greater than $k-2$, degree of $q$ is not greater than $k-1$, the $x$-coordinates of singular points of $H$ are roots of $q$ and

$$
p(x)\left(3 H(x, y)-y H_{y}(x, y)\right)=q(x) H_{x} .
$$

The deformation $F$ is obtained from $H$ by shifting a singular point to the origin and blowing up. Both operations preserve the above relations. Note that the quasidegree of $y$ is decreasing by one and qdeg $f=3 k-2$.

Substituting

$$
\begin{gathered}
q(x)=x q_{1}(x), \quad H(x, x y)=x^{2} F(x, y), \\
H_{x}(x, x y)=2 x F(x, y)+x^{2} F_{x}(x, y)-x y F_{y}(x, y), \quad H_{y}(x, x y)=x F_{y}(x, y),
\end{gathered}
$$

we obtain

$$
\begin{aligned}
W_{k}(x, x y)=x^{k}\left(3 x^{2} F(x, y)-x^{2} y F_{y}(x, y)\right) & \\
& -r_{k}(x)\left(2 x F(x, y)+x^{2} F_{x}(x, y)-x y F_{y}(x, y)\right) .
\end{aligned}
$$

The right-hand side can be divided by $x$. Thus $W_{k}(x, x y) / x$ is an underdiagonal polynomial (its quasidegree is less than $3 k-2$ ).

In such a way we show that for $k=0,1, \ldots,\left(3 x^{k+1}-2 r_{k}(x)\right) F(x, y)$ is equivalent to an underdiagonal polynomial modulo the gradient ideal. Since the same is true for $F$ itself (consider $\left.(3 k-2) F-(k-1) y F_{y}-x F_{x}\right)$, we get that the class of any $x^{k} F$ is underdiagonal.

From the second relation we obtain

$$
p(x)\left(3 x^{2} F(x, y)-x^{2} y F_{y}(x, y)\right)=x q_{1}(x)\left(2 x F(x, y)+x^{2} F_{x}(x, y)-x y F_{y}(x, y)\right) .
$$

We divide both sides by $x^{2}$ and deduce that

$$
\left(3 p(x)-2 q_{1}(x)\right) F(x, y)
$$

is equivalent to 0 in the factor algebra. Since $\operatorname{deg}\left(3 p(x)-2 q_{1}(x)\right)<k-1$, the polynomials $y F, x y F, \ldots$ and the underdiagonal base elements do not generate the whole factor algebra. Indeed, the dimension of the subspace spanned by $y F, x y F, \ldots$ is smaller than the number of diagonal and overdiagonal base elements.

To finish the proof we show that $y^{2} F$ is also equivalent to underdiagonal polynomial. This follows from the above and the following relations:
A: $\left(3 y^{2}+e x^{2 m-1} d(x)^{2}\right) F=\left(y^{3}+e x^{2 m-1} d(x)^{2} y+x^{3 m-2} d(x)^{3}\right) F_{y}$,
B: $\left(y^{2}+\left(x^{m-1} b(x)\right)^{\prime}\right) F=\left(x y^{2}+x^{m-1} b(x)\right) F_{x}$,
$\mathrm{C}: m>2, \quad 3 y^{2} F=\left(y+x^{m-3} c(x)\right) F_{y}$,
D: $m=2, \quad 3 y^{2} F=(y+(c(x)-c(0)) / x) F_{y}+c(0) y^{2}$. 


\section{2. $Q_{k, 0}$ case}

LEMMA 9. The point $\lambda$ belongs to the versal discriminant if and only if $G_{\lambda}$ is of type $A, B, C$ or $D$ and respectively the polynomial $x d(x), x b(x), x c(x)$ or $x\left(x c_{1}(x)-a^{2}\right)$ has at most $k-1$ different roots.

Proof. In $Q_{k, 0}$ case we have to consider the following polynomials: $F_{\lambda}, x F_{\lambda}, \ldots$, $\ldots, x^{m} F_{\lambda}, \ldots, y F_{\lambda}, x y F_{\lambda}, \ldots, x^{m} y F_{\lambda}, \ldots, z F_{\lambda}$ and $z y F_{\lambda}$.

Cases A, B, C. We repeat the same arguments as above. The deformation $G$ is obtained from $F$ by stabilization and blowing up. Note that the quasidegree of $z$ is decreasing by one and qdeg $g=3 k-3$.

We substitute

$$
\begin{gathered}
F(x, y)=x G(x, y, z)-\frac{1}{2} z G_{z}(x, y, z), \quad F_{y}(x, y)=x G_{y}(x, y, z), \\
F_{x}(x, y)=G(x, y, z)+x G_{x}(x, y, z)-z G_{z}(x, y, z)
\end{gathered}
$$

and obtain

$$
\begin{aligned}
W_{k}(x, x y) & =x^{k}\left(3 x^{3} G(x, y, z)-x^{3} y G_{y}(x, y, z)-\frac{3}{2} x^{3} z G_{z}(x, y, z)\right) \\
- & r_{k}(x)\left(3 x^{2} G(x, y, z)+x^{3} G_{x}(x, y, z)-x^{2} y G_{y}(x, y, z)-2 x^{2} z G_{z}(x, y, z)\right) .
\end{aligned}
$$

The right-hand side can be divided by $x^{2}$. Thus $x^{-2} W_{k}(x, x y)$ is an underdiagonal polynomial (qdeg $<3 k-3$ ).

In such a way we show that for $k=0,1, \ldots,\left(x^{k+1}-r_{k}(x)\right) G(x, y, z)$ is equivalent to an underdiagonal polynomial modulo the gradient ideal. Since the same is true for $G$ itself (consider $\left.(3 k-3) G-(k-1) y G_{y}-x G_{x}-(3 / 2 k-1) z G_{z}\right)$, we get that the class of any $x^{k} G$ is underdiagonal.

From the second relation we obtain:

$$
\begin{aligned}
p(x)(3 & \left.x^{3} G(x, y, z)-x^{3} y G_{y}(x, y, z)-\frac{3}{2} x^{3} z G_{z}(x, y, z)\right) \\
& =x q_{1}(x)\left(3 x^{2} G(x, y, z)+x^{3} G_{x}(x, y, z)-x^{2} y G_{y}(x, y, z)-2 x^{2} z G_{z}(x, y, z)\right) .
\end{aligned}
$$

We divide both sides by $x^{3}$ and deduce that

$$
\left(p(x)-q_{1}(x)\right) G(x, y)
$$

is equivalent to 0 in the factor algebra. Since $\operatorname{deg}\left(p(x)-q_{1}(x)\right)<k-1$, the polynomials $y G, x y G, \ldots$ and the underdiagonal base elements do not generate the whole factor algebra. Indeed, the dimension of the subspace spanned by $y G, x y G, \ldots$ is smaller than the number of diagonal and overdiagonal base elements.

To finish the proof it is enough to notice that since $z x$ belongs to the gradient ideal, $z G$ and $z y G$ are equivalent to underdiagonal polynomials.

Case D. In this case we have

$$
\begin{gathered}
G=x z^{2}+2 a z+y^{3}+c_{1}(x), \\
G_{y}=3 y^{2}, \quad G_{x}=z^{2}+c_{1}^{\prime}(x), \quad G_{z}=2 x z+2 a .
\end{gathered}
$$

We put

$$
\begin{gathered}
\tilde{G}=G-z G_{z}-\frac{1}{3} y G_{y}+x G_{x}=x c_{1}^{\prime}(x)+c_{1}(x), \\
D=x^{2} G_{x}-\frac{1}{2}(x z-a) G_{z}=x^{2} c_{1}^{\prime}(x)+a^{2} .
\end{gathered}
$$


Since $\operatorname{deg} D=3 k-2$, using $D, G_{x}$ and $G_{z}$ we show that for any nonnegative $k x^{k} \tilde{G}$ is equivalent to an underdiagonal polynomial.

Furthermore let $d_{1}(x)$ be the greatest common divisor of $x c_{1}(x)-a^{2}$ and its derivative $x c_{1}^{\prime}(x)+c_{1}(x)$. We put $x c_{1}^{\prime}(x)+c_{1}(x)=d_{0} d_{1}$ and $x c_{1}^{\prime}(x)=d_{2} d_{1}$. Then

$$
\left(d_{2}-x d_{0}\right) \tilde{G}=\left(d_{2}-x d_{0}\right) d_{0} d_{1}=d_{0} D_{1} .
$$

Hence if the degree of $\left(d_{2}-x d_{0}\right)$ is smaller than $k-1$ then the polynomials $y \tilde{G}, x y \tilde{G}, \ldots$ and the underdiagonal base elements do not generate the whole factor algebra.

To finish the proof it is enough to notice that $z$ modulo gradient ideal is equivalent to the polynomial $x c_{1}^{\prime}(x) / a$. Thus $z G$ and $z y G$ belong in the factor algebra to subspaces spanned by $x^{k} \tilde{G}$ and $y x^{k} \tilde{G}$.

\section{References}

[1] V. I. Arnol'd, S. M. Guseŭn-Zade and A. N. Varchenko, Singularities of Differentiable Maps, vol. 1, Birkhäuser, Boston, 1985.

[2] J. Damon, On the Pham example and the universal topological stratification of singularities, in: Singularities, Banach Center Publ. 20, PWN-Polish Scientific Publishers, Warszawa, 1988, 161-167.

[3] J. Damon, A-equivalence and the equivalence of sections of images and discriminants, in: Singularity Theory and its Applications, Part 1 (Coventry 1988/1989), Lecture Notes in Math. 1492, Springer, Berlin, 1991, 93-121.

[4] J. Damon, A. Galligo, Universal topological stratification for the Pham example, Bull. Soc. Math. France 121 (1993), 153-181.

[5] R. Hartshorne, Algebraic Geometry, Graduate Texts in Math. 52, Springer, New York, 1977.

[6] P. Jaworski, Decompositions of hypersurface singularities of type $J_{k, 0}$, Ann. Polon. Math. 59 (1994), 117-131.

[7] P. Jaworski, On the versal discriminant of the $J_{k, 0}$ singularities, Ann. Polon. Math. 63 (1996), 89-99.

[8] E. Looijenga, Semi-universal deformation of a simple elliptic hypersurface singularity, I: Unimodularity, Topology 16 (1977), 257-262.

[9] A. du Plessis, C. T. C. Wall, Topological stability, in: Singularities (Lille, 1991), London Math. Soc. Lecture Note Ser. 201, Cambridge Univ. Press, Cambridge, 1994, 351-362.

[10] A. du Plessis, C. T. C. Wall, The Geometry of Topological Stability, London Math. Soc. Monogr. (N.S.) 9, Oxford Sci. Publ., Oxford Univ. Press, New York, 1995.

[11] K. Wirthmüller, Universell topologische triviale Deformationen, Ph.D. thesis, University of Regensburg, 1979. 